\title{
EDITORIAL
}

\section{Do Ulcers Heal Structurally and Functionally?}

\author{
Susan J. Hagen ${ }^{1}$ \\ Published online: 9 November 2019 \\ (c) Springer Science+Business Media, LLC, part of Springer Nature 2019
}

The gastric mucosa is constantly exposed to a harsh environment due to numerous factors, including abrasion from consumed food, damage from orally administered drugs, digestive factors produced by mucosal epithelial cells including gastric acid and pepsinogen, ischemia and other stressors, and infection with Helicobacter pylori. To meet these daunting challenges, the stomach has numerous mucosal defense mechanisms that prevent mucosal damage and maintain mucosal integrity [1]. Mucosal injury occurs when damaging factors overwhelm defensive factors or when mucosal defense mechanisms, per se, are impaired [1]. Gastric acid hypersecretion, the repeated ingestion of high saltcontaining foods or nonsteroidal anti-inflammatory drugs (NSAIDs), and/or H. pylori infection can result in superficial injury or deep ulceration of the mucosa manifest as erosions or peptic ulcers that usually heal after the insult is removed. Despite the decreasing incidence of peptic ulcers in the past 20 years, largely attributed to the use of proton pump inhibitors to inhibit acid secretion and the eradication of H. pylori, peptic ulcers are still diagnosed in $5-10 \%$ of the general population [2, 3]. Furthermore, even after treatment for $H$. pylori infection, there is a cumulative $20 \%$ probability of ulcer recurrence within 6 months [4] with the 5-year cumulative probability of ulcer recurrence in the absence of $H$. pylori infection $>35 \%$ [5]. Despite active work to understand factors that facilitate the initiation of or protection against ulceration, mechanisms that regulate the recurrence (relapse) of previously healed ulcers are largely unknown.

One idea concerning ulcer relapse is that a healed ulcer does not regain its original architecture that includes important mucosal protective functions. Animal models, particularly the acetic acid model of ulcer induction [6], have provided considerable insight into the issue of ulcer relapse. While complete repair after ulcer induction was

Susan J. Hagen

shagen@bidmc.harvard.edu

1 Department of Surgery/Division of General Surgery, Beth Israel Deaconess Medical Center, Harvard Medical School, E/RW-871, 330 Brookline Avenue, Boston, MA 02115, USA observed in other animal models of ulcer induction, ulcer relapse $>100$ days after initial ulcer induction is routinely observed in the acetic acid model, similar to the timing of ulcer relapse in humans [6]. Using scanning electron microscopy, investigators observed that the epithelium surrounding the ulcerated area was damaged, which was thought to represent the initial step in ulcer relapse [6]. Over time, this initial observation was further examined experimentally, particularly with histopathology, with the results from numerous labs suggesting that the regenerated epithelium after ulcer healing was abnormal, leading to the notion that a "healed" ulcer is at greater risk of subsequent damage. Recent studies by Aihara et al. [7], using mouse models with acetic acidinduced ulceration and sophisticated genomic and immunohistochemical analysis, determined that the regenerated mucosa after ulceration remains abnormal for months after the initial injury. Specifically, their study demonstrated that the regenerated mucosa showed: (1) an increase in epithelial stem cell/progenitor cell number that was associated with the lack of differentiated cell types including parietal and chief cells for up to 8 months; (2) impaired expression of the sodium hydrogen exchanger 2 (NHE2, gene Slc9A2), which is required to assist with trefoil factor (Tff2, gene Tff2)2-dependent mucosal repair after injury in the stomach; and (3) mucous metaplasia that resembles spasmolytic polypeptide-expressing metaplasia (SPEM), a precancerous condition [7]. Consistent with the abnormal epithelial profile and lack of tissue homeostasis in the ulcerated mucosa, subsequent infection with $H$. pylori induced ulcer relapse and impaired healing [7]. Results from this study concluded that the stomach mucosa in mice takes up to 8 months to fully heal, which is half of the lifetime of a mouse, and the ulcerated area is prone to further injury unless the insulting environment is mitigated [7].

Perhaps even more important than structural repair after ulceration would be whether or not normal mucosal function returns in the ulcerated area during healing. In this issue of Digestive Diseases and Sciences, Matthis et al. [8] provide important new insight into mucosal functions that are not restored in the ulcerated mucosa until 8 months after 
the initial ulceration in conjunction with structural repair of the mucosa. Furthermore, with repeat injury, recovery of mucosal ion transport is impaired, likely due in part to dysregulation of intracellular $\mathrm{pH}\left(\mathrm{pH}_{\mathrm{i}}\right)$ regulation in the restituting cells.

The normal gastric mucosa utilizes ion transport mechanisms to drive acid secretion and activate mucosal protective functions against gastric acid including mucosal bicarbonate secretion and the regulation of $\mathrm{pH}_{\mathrm{i}}$. During mucosal repair, similar ion transport mechanisms are important for restitution after superficial injury, particularly sodium-driven transporters that are important for mucosal restitution as originally determined using Ussing chambered bullfrog stomach mucosa [9]. Although mounting mouse tissues in Ussing chambers is a considerable technical challenge, the work by Matthis et al. [8] uses this technique beautifully to evaluate levels of active ion transport in healed ulcerated mucosa over time and in healed ulcerated mucosa treated with hyperosmolar $\mathrm{NaCl}$ to induce superficial epithelial damage as a repeated insult. These studies utilized the power of genetically modified mice to explore the contributions of Tff 2 and NHE2 to gastric mucosal active ion transport after superficial injury. To evaluate the functions of $\mathrm{Na}^{+}$-driven transporters, the group used gastroid preparations in vitro with small laser wounds in conjunction with live-cell confocal microscopy and specific dyes and inhibitors. In particular, they focused on NHE transporters and their regulation of $\mathrm{pH}_{\mathrm{i}}$ during wound repair.

The first important result of this study clearly showed significant differences in repair characteristics of the healing mucosa. At 30 days after ulceration, there was a general lack of active ion transport recovery after $\mathrm{NaCl}$ injury in this model. Since parietal cells, the major drivers of active ion transport in stomach mucosa [10] are not present in the healing mucosa at this time point, the investigators blocked acid secretion to mitigate this important difference in the mucosal samples. In tissues 8 months after ulceration, variability was considerable and as such, no differences in recovery were reported. The data, however, suggest that since inter-animal variability in the reconstitution of the ulcerated tissue was high, increasing the number of mice used at this later time point would likely be needed to provide the most robust results.

Since this group originally described the relationship between Tff 2 and NHE2 in mucosal repair following gastric injury, they probed the contributions of these proteins to ion transport function following $\mathrm{NaCl}$ injury in healed tissue after ulceration. In 30 day healed ulcers induced in Tff2-deficient mice, regeneration was inhibited compared with control mice. As might be expected, Tff2-deficient mice with or without ulceration showed a similar response to $\mathrm{NaCl}$ injury. This result was consistent with previous data from this group and others, demonstrating the important contributions of Tff 2 toward mucosal repair after injury. To complete the story, the group attempted to use Slc9A2-deficient mice to confirm that blocking either pathway in healing mucosa impairs repair after $\mathrm{NaCl}$ injury. This was an important aspect of the study because Aihara et al. [7] showed that the mRNA for $S l c 9 A 2$ is highly downregulated in healing ulcers, whereas Tff2 is highly upregulated; because they function together, blockade of NHE2 expression should mitigate the repair-promoting function of Tff 2 in the healing ulcer. Unfortunately, Slc9A2-deficient mice were bred on a genetic background that did not promote repair after $\mathrm{NaCl}$ injury. Thus, to answer the question, specific drug antagonists of NHE2 activity were used to demonstrate impaired repair after $\mathrm{NaCl}$ injury occurs after blockade of NHE2. It was interesting that the group studied repair only in control mice, which were wild-type C57BL6/J mice subjected to $\mathrm{NaCl}$ injury, but did not evaluate 30 day or 8 month ulcerated tissues using the same methods. Due to this issue, the time at which attenuated NHE2 function contributes to the repair of active ion transport after $\mathrm{NaCl}$ injury in the healed mucosa remains unclear. Nevertheless, data using organoids with single cell damage in the presence of NHE antagonists clearly showed that NHE is involved in cell migration by regulating $\mathrm{pH}_{\mathrm{i}}$ of the migrating cells. Although organoids provided outstanding and consistently reproducible data, in vivo correlation in mouse tissues was lacking.

The general premise of this work is very interesting, clearly demonstrating deficits in active ion transport in healed mucosa that is subjected to subsequent injury. Although NHE2/Tff2 make important contributions toward mucosal repair after gastric injury, the studies in this paper do not improve understanding of their role in facilitating an electrogenic response in repaired mucosa after injury. As noted by the authors, since NHE is an electroneutral exchanger incapable of generating a transmucosal current, NHE2 must be involved indirectly in the normal repair response after injury in ways that remain unknown. Numerous other electrogenic pathways exist in the stomach that are responsible for maintaining a functional transmucosal $\mathrm{Na}^{+}$ gradient and are likely involved in $I_{\mathrm{SC}}$ recovery after hypertonic $\mathrm{NaCl}$-induced injury. With the outstanding model and demonstrated ability to create and study ulcerated gastric mucosa in genetically modified mice, the work in this article sets the stage for exciting new studies to dissect mechanisms leading to early ulcer relapse in animal models. Understanding the etiology of ulcer relapse is important and will ultimately enable the development of therapeutic interventions aimed at reducing the burden of peptic ulcer disease. 


\section{References}

1. Laine L, Takeuchi K, Tarnawski AS. Gastric mucosal defense and cytoprotection: bench to bedside. Gastroenterology. 2008; 135:41-60.

2. Lanas A, Chan FKL. Peptic ulcer disease. Lancet. 2017;390:613-624.

3. Everhart JE. The burden of digestive diseases in the United States. US Government Printing Office. 2008; NIH Publication No. 09-6443:97-106.

4. Laine L, Hopkins RJ, Girardi LS. Has the impact of Helicobacter pylori therapy on ulcer recurrence in the United States been overstated? A meta-analysis of rigorously designed trials. Am J Gastroenterol. 1998;93:1409-1415.

5. Seo JH, Hong SJ, Kim JH, et al. Long-term recurrence rates of peptic ulcers without Helicobacter pylori. Gut Liver. 2016;10:719-725.

6. Okabe S, Amagase K. An overview of acetic acid ulcer modelsthe history and state of the art of peptic ulcer research. Biol Pharm Bull. 2005;28:1321-1341.
7. Aihara E, Matthis AL, Karns RA, et al. Epithelial regeneration after gastric mucosal ulceration causes prolonged cell-type alterations. Cell Mol Gastroenterol Hepatol. 2016;2:625-647.

8. Matthis AL, Kaji I, Engevik KA, et al. Deficient active transport activity in healing mucosa after mild gastric epithelial damage. Dig Dis Sci. (Epub ahead of print). https://doi.org/10.1007/s1062 0-019-05825-x.

9. Hagen SJ, Morrison SW, Law CS, Yang DX. Restitution of the bullfrog gastric mucosa is dependent on a DIDS-inhibitable pathway not related to $\mathrm{HCO}_{3}{ }^{-}$ion transport. Am J Physiol Gastrointest Liver Physiol. 2004;286:G596-G605.

10. Carothers JM, Forte JG, Machen TE. Computer modeling of the gastric parietal cell: significance of the canalicular space, gland lumen, and variable canalicular $\left[\mathrm{K}^{+}\right]$. Am J Physiol Gastrointest Liver Physiol. 2018;310:G671-G681.

Publisher's Note Springer Nature remains neutral with regard to jurisdictional claims in published maps and institutional affiliations. 